\title{
A matter of fat: protecting the injured heart
}

\author{
Daan Paget, Naveed Akbar \\ Division of Cardiovascular Medicine, Radcliffe Department of Medicine, University of Oxford, Oxford, UK \\ Correspondence to: Dr. Naveed Akbar. Division of Cardiovascular Medicine, Radcliffe Department of Medicine, University of Oxford, Oxford OX3 \\ 9DU, UK. Email: Naveed.Akbar@Cardiov.ox.ac.uk. \\ Comment on: Crewe C, Funcke JB, Li S, et al. Extracellular vesicle-based interorgan transport of mitochondria from energetically stressed adipocytes. \\ Cell Metab 2021;33:1853-1868.e11.
}

Received: 30 November 2021; Accepted: 27 December 2021; Published: 28 February 2022.

doi: 10.21037/exrna-21-28

View this article at: https://dx.doi.org/10.21037/exrna-21-28

\section{Introduction}

Adipose tissue was long thought to be an inert bodily substance, which was filled with lipid and surrounded the peripheral organs for storage of excess energy and acted as a buffer for mechanical strain. However, under normal physiological conditions adipose tissue performs important functions by secretion of adipokines and growth factors to regulate of lipid metabolism (1). These adipocyte processes are tightly regulated by homeostatic mechanisms and neighbouring cell-to-cell interactions, which maintains body temperature by consuming fatty acids via nonshivering thermogenesis. Adipose tissue experiences profound maladaptation in response to calorie excess, such as in obesity, which induces adipose tissue hypertrophy, dysregulates mitochondrial biogenesis, lowers mitochondrial mass and favours oxidative phosphorylation. Furthermore, the type and anatomical location of adiposity drives diverse regional effects in adipose tissue biology, which are reflected in transcriptomic and proteomic profiles $(2,3)$ and independently elevate metabolic and cardiovascular disease risk for major events such as myocardial infarction (4).

Yet, surprisingly, a complex relationship exists between adiposity and the high prevalence of cardiometabolic diseases. It's well established that perivascular adipose tissue can drive atherosclerosis (5) and is independently associated with residual cardiovascular risk in patients (4). Vascular cells also exert powerful effects on neighbouring adipose tissue (6). By contrast, epidemiological data show that overweight individuals suffering from chronic inflammatory diseases do better than normal-weight individuals with the same chronic disease (7). However, the underlying factors which drive these adipose tissue survival benefits remain elusive. One potential mechanism that may explain the survival paradox could be via physiologically stressed adipose tissue. Chronic high fat feeding in mice is a model of diet-induced obesity, which results in oxidative stress, lowers mitochondrial mass, cellular ATP and induces mitochondrial dysfunction, similar to mitochondrial dysfunction observed in metabolic disease patients. However, not all mitochondrial stress is pathological, transient mild stresses confer protection through mitohormesis; a process where mitochondrial derived reactive oxygen species can lead to a persistent adaptation and protection against future stressors (8).

High fat diet feeding in mice results in numerous multi organs changes. To discretely study the role of adipocyteinduced stress on the myocardium Crewe et al. used an inducible mouse model, which selectively overexpresses mitochondrial ferritin (FtMT) (adipo-FtMT mouse) following doxycycline exposure. This results in systemic glucose intolerance, low adiponectin levels and oxidative damage in adipocytes, that mimic high fat feeding (9). Interestingly, adipo-FtMT mice show enhanced $\beta$-cell mass, suggestive of beneficial interorgan signalling. Nevertheless, high fat feeding in mice and obesity in humans induces a number of systemic pathological organ manifestations, not least cardiac dysfunction by enhanced reactive oxygens species and an elevated risk for subsequent myocardial infarction. Adipo-FtMT mice show similar levels of cardiac tissue mitochondrial $\mathrm{H}_{2} \mathrm{O}_{2}$ to that observed in wild-type mice after 16 weeks on a high fat diet (9). Thus, establishing that localised adipocyte stress induces across organ dysfunction in the myocardium.

To determine the mode of across-organ communication 


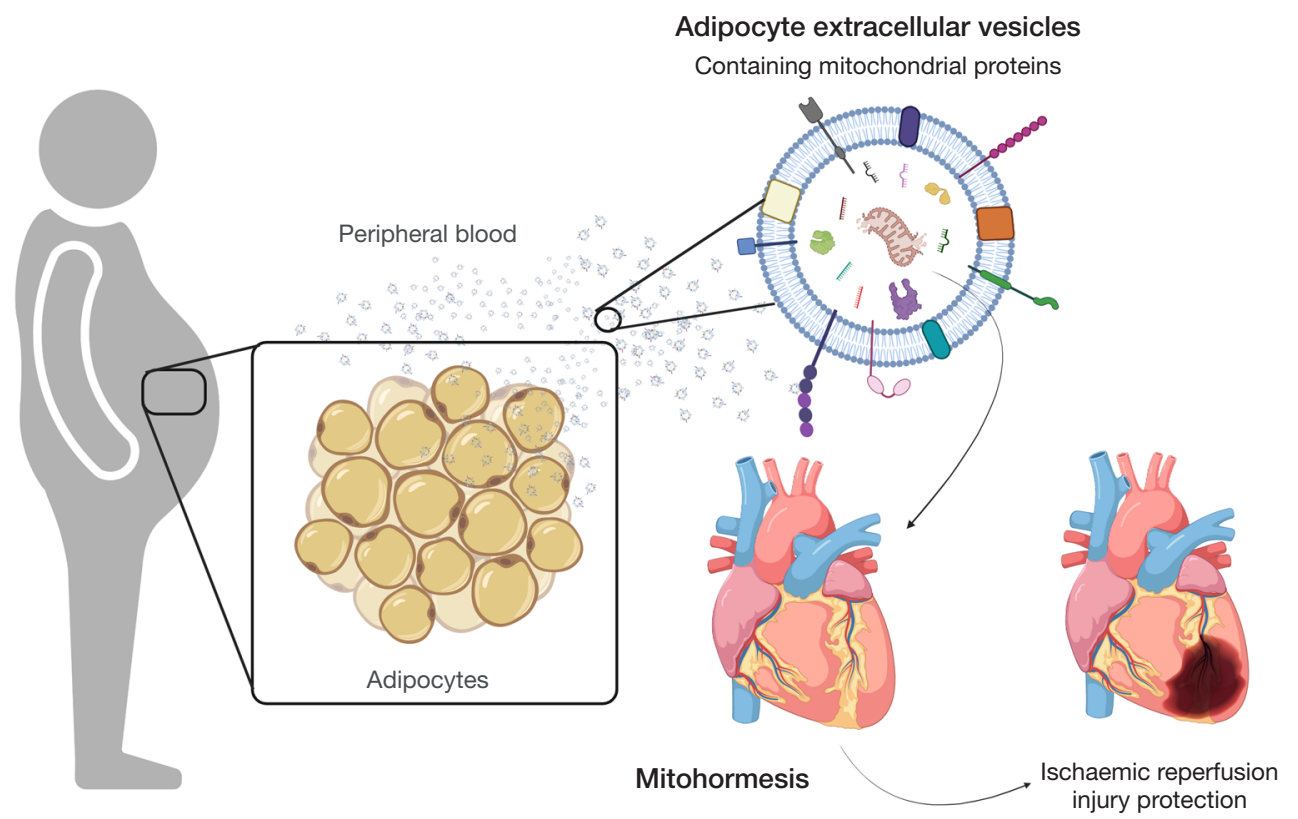

Figure 1 Crewe et al. 2021 described how physiologically stressed adipocytes release extracellular vesicles, that contain mitochondrial proteins and induce pro-oxidation mitohormesis in the heart and protect against future ischaemic reperfusion injury.

in adipo-FtMT mice on a doxycycline enriched high fat diet, Crewe et al. investigated circulating circulating extracellular vesicles (EV). Long range organ-to-organ and organ-to-tissue signalling in this context can be mediated by EV (10). EV are bi-lipid enclosed envelopes encompassing, apoptotic bodies, microvesicles and exosomes. Adipocytes $\mathrm{EV}$ under normal physiological conditions contribute to the circulating plasma pool (10). Following pathological stimuli, such as perturbations in glucose, inflammation, or in patients with underlying cardiovascular disease and metabolic disease, adipocyte EV show differential enrichment of EV-cargo, including proteins, microRNAs and lipids (11). Pathological adipocyte EV regulate properties in recipient cells, including alterations in cellular transcription distally from their source in other organs, with potential to accumulate in the myocardium (10).

Crewe et al. demonstrate how high fat feeding in mice augments adipocyte $\mathrm{EV}$ release and enriches functional but oxidatively damaged mitochondria within their membranes, that confer mitohormesis and protect against subsequent reperfusion injury in mice (8) (Figure 1). Circulating mouse adipo-FtMT were positive for generalised $\mathrm{EV}$-associated proteins ALG-2-interacting protein X (ALIX), CD63, CD81 and contained more fatty acid binding protein-4 (FABP4), that has previously been shown to be present in adipocyte derived EV (12). The results from this study are similar to other reports of alterations in blood EV number in models of diet-induced obesity (13). There are earlier description of mitochondria and mitochondrial derived proteins within $\mathrm{EV}$ from activated platelets and stem cells $(14,15)$. But numerous studies, including investigations from our laboratory utilise mitochondrial proteins to demonstrate the absence of cellular contaminants in isolated $\mathrm{EV}$ preparations in line with reporting guidelines by the International Society for Extracellular Vesicles (ISEV) (16). It's important to note that many investigators may be selecting against functionally important vesicles populations during protocol optimisations, which contain functional mitochondria and mitochondrial proteins based on these recommendations.

Adipo-FtMT serum EV accumulate in the heart in vivo and mediate pro-oxidant effects, but the authors note that the heart is not the predominant site of accumulation in vivo, with significant accumulation in the liver. However, the authors do not allude to other dominant sites of EV capture, such as the spleen, lung and kidney, which are also liable to metabolic dysfunction and show mitochondrial stress in chronic diseases (17). The primary cell type for $\mathrm{EV}$ capture in numerous organs are resident macrophages. EV enriched with mitochondrial proteins can modulate pro-inflammatory and anti-inflammatory responses in macrophages $(14,15)$. The present study 
reports that adipocyte $\mathrm{EV}$, in part, are capable of evading the mononuclear phagocytic system and induce localised effects in the myocardium (8). But the EV-properties, which orchestrate this adipocyte-to-myocardium signalling axis are not understood. Determination of biodistribution of EV in vivo is complex, owing to their very small size. Lipophilic dyes, such as PKH26 used in this study, to exogenously label $\mathrm{EV}$ and subsequently inject them into mice is one method to track EV fate post-mortem. However, lipophilic dye labelling of $\mathrm{EV}$ for biodistribution in vivo has a number of limitations, including non-specific labelling of lipoproteins, dye leaching and formation of dye aggregates, which have been discussed in detail elsewhere (18). These highlight the need for more in-depth EV tracking analyses of adipocyte derived $\mathrm{EV}$ in vivo, determination of the $\mathrm{EV}$ characteristics that allows localised capture to the myocardium and establishment of whether adipocyte EV capture in other organs confers mitohormesis.

The effective dose injected represented as $0.033 \%$ of endogenous circulating $\mathrm{EV}$ in the mouse, suggests very powerful effects on redox signalling. Numerous studies utilise doses, which are several orders a magnitude greater than those used here, or present under normal physiological or pathological conditions (19). But it remains uncertain, which proportion of the injected $\mathrm{EV}$ accumulated within the myocardium versus those, which localise to other tissues such as the liver, which outweigh the mass of the myocardium. Cardiomyocytes, are a predominant cell type in the heart, uptake adipocyte EV and adipo-FtMT high fat diet fed serum EV induce oxidative damage in cardiomyocytes when compared to serum EV from controls (8). Investigations by others suggest that uptake of $\mathrm{EV}$ by other cell types found in the myocardium surpass cardiomyocyte EV uptake, namely EV uptake by vascular endothelial cells is far greater than cardiomyocytes and even fibroblasts. Endothelial cell dysfunction is a hallmark event in metabolic and cardiovascular dysfunction and the authors of the present study have previously reported the transfer of caveolin-1 from endothelial cells to adipocytes in EV. Highlighting the intricate complexity of EV mediated signalling in whole organisms and across organs systems (20).

To study cell-to-cell specific EV communication pathways, cell-specific EV inhibition is required. As of now, it is not possible to completely abolish EV mediated signalling in distinct cell types, but $\mathrm{EV}$ release in vivo can be limited through application of GW4869, a neutral sphingomyelinase 2 (nSMase2) inhibitor of ceramides. Circulating plasma ceramides and plasma EV-ceramides regulate vascular redox signalling and influence outcomes in patients with cardiovascular disease (11). GW4869 is general inhibitor of nSMase2, and it is likely that numerous processes dependent on ceramide synthesis will be affected if such inhibitors are used for longer periods of time, which is often necessary for treatment of chronic diseases such as cardiometabolic dysfunction. Cell-specific adipocyte derived EV inhibitors will enable delineation of adipocyte $\mathrm{EV}$ versus those generated by other cell types in vivo.

Emerging data shows that the heart can also produce signals to influence body weight, through systemic regulation of energy metabolism and mitochondrial biogenesis (21). Specifically, plasma EV isolated following ischemic reperfusion injury induce adipocyte endoplasmic reticulum stress through an EV-miRNA cluster, which can similarly to this study, be inhibited by application of GW4869 (22).

The findings by Crewe et al. have important health implications. But subsequent investigations will need to determine, whether the anatomical location of adipose tissue has negative consequences on metabolic health. Visceral white adipose tissue is more pathological than subcutaneous white adipose tissue. These adipose tissue depot differences may have important variations in their ability to mediate mitohormesis through EV mediated signalling, which may be further influenced by sex. Males are more likely to store visceral adipose tissue, whereas women are more likely to preference subcutaneous adipose tissue, which are driven by sex hormones (23).

The study highlights the tremendous complexity of across organ signalling, diverging away from a narrow focus on immediate cell-to-cell interactions locally in tissue microenvironments. Across organ communication in pathological settings may be both acute, with selective bursts of $\mathrm{EV}$ for immediate effects and prolonged in chronic disease. A better understanding of how EV mediated organto-organ communication mediates pathological signalling will underpin the creation of new blood based diagnostic tests, to offer precision medicine and potentially EV-based therapeutics to protect tissues from future insults.

\section{Acknowledgments}

Funding: This work was supported by research grants from the British Heart Foundation (BHF) Centre of Research Excellence, Oxford (RE/13/1/30181 and RE/18/3/34214 to NA); British Heart Foundation Project Grant (PG/18/53/33895 to NA); Nuffield Benefaction for 
Medicine and the Wellcome Institutional Strategic Support Fund (ISSF) and a Health Research Bridging Salary Scheme (HRBSS) to NA.

\section{Footnote}

Provenance and Peer Review: This article was commissioned by the editorial office, ExRNA. The article has undergone external peer review.

Conflicts of Interest: Both authors have completed the ICMJE uniform disclosure form (available at https:// exrna.amegroups.com/article/view/10.21037/exrna-21-28/ coif). DP reports funding from Clarendon Fund, Medical Research Council Oxford and Lady Margaret Hall, Oxford. All above have no conflict of interest with the article. NA reports funding from British Heart Foundation (BHF) Centre of Research Excellence, Oxford (RE/13/1/30181 and RE/18/3/34214 to NA via institution), British Heart Foundation Project Grant (PG/18/53/33895 to NA via institution), Nuffield Benefaction for Medicine and the Wellcome Institutional Strategic Support Fund (ISSF) (to NA via institution), and Health Research Bridging Salary Scheme (HRBSS) (to NA via institution). All above have no conflicts of interest with the article. The authors have no other conflicts of interest to declare.

Ethical Statement: The authors are accountable for all aspects of the work in ensuring that questions related to the accuracy or integrity of any part of the work are appropriately investigated and resolved.

Open Access Statement: This is an Open Access article distributed in accordance with the Creative Commons Attribution-NonCommercial-NoDerivs 4.0 International License (CC BY-NC-ND 4.0), which permits the noncommercial replication and distribution of the article with the strict proviso that no changes or edits are made and the original work is properly cited (including links to both the formal publication through the relevant DOI and the license). See: https://creativecommons.org/licenses/by-nc-nd/4.0/.

\section{References}

1. Kahn CR, Wang G, Lee KY. Altered adipose tissue and adipocyte function in the pathogenesis of metabolic syndrome. J Clin Invest 2019;129:3990-4000.

2. Bäckdahl J, Franzén L, Massier L, et al. Spatial mapping reveals human adipocyte subpopulations with distinct sensitivities to insulin. Cell Metab 2021;33:2301.

3. Raajendiran A, Krisp C, Souza DP, et al. Proteome analysis of human adipocytes identifies depot-specific heterogeneity at metabolic control points. Am J Physiol Endocrinol Metab 2021;320:E1068-84.

4. Mazzotta C, Basu S, Gower AC, et al. Perivascular Adipose Tissue Inflammation in Ischemic Heart Disease. Arterioscler Thromb Vasc Biol 2021;41:1239-50.

5. Ahmadieh S, Kim HW, Weintraub NL. Potential role of perivascular adipose tissue in modulating atherosclerosis. Clin Sci (Lond) 2020;134:3-13.

6. Herold J, Kalucka J. Angiogenesis in Adipose Tissue: The Interplay Between Adipose and Endothelial Cells. Front Physiol 2021;11:624903.

7. Oikonomou EK, Antoniades C. The role of adipose tissue in cardiovascular health and disease. Nat Rev Cardiol 2019;16:83-99.

8. Crewe C, Funcke JB, Li S, et al. Extracellular vesicle-based interorgan transport of mitochondria from energetically stressed adipocytes. Cell Metab 2021;33:1853-1868.e11.

9. Kusminski CM, Ghaben AL, Morley TS, et al. A Novel Model of Diabetic Complications: Adipocyte Mitochondrial Dysfunction Triggers Massive $\beta$-Cell Hyperplasia. Diabetes 2020;69:313-30.

10. Akbar N, Azzimato V, Choudhury RP, et al. Extracellular vesicles in metabolic disease. Diabetologia 2019;62:2179-87.

11. Akawi N, Checa A, Antonopoulos AS, et al. Fat-Secreted Ceramides Regulate Vascular Redox State and Influence Outcomes in Patients With Cardiovascular Disease. J Am Coll Cardiol 2021;77:2494-513.

12. Eguchi A, Lazic M, Armando AM, et al. Circulating adipocyte-derived extracellular vesicles are novel markers of metabolic stress. J Mol Med (Berl) 2016;94:1241-53.

13. Kumar A, Sundaram K, Mu J, et al. High-fat diet-induced upregulation of exosomal phosphatidylcholine contributes to insulin resistance. Nat Commun 2021;12:213.

14. Phinney DG, Di Giuseppe M, Njah J, et al. Mesenchymal stem cells use extracellular vesicles to outsource mitophagy and shuttle microRNAs. Nat Commun 2015;6:8472.

15. Boudreau LH, Duchez AC, Cloutier N, et al. Platelets release mitochondria serving as substrate for bactericidal group IIA-secreted phospholipase A2 to promote inflammation. Blood 2014;124:2173-83.

16. Théry C, Witwer KW, Aikawa E, et al. Minimal information for studies of extracellular vesicles 2018 (MISEV2018): a position statement of the International 
Society for Extracellular Vesicles and update of the MISEV2014 guidelines. J Extracell Vesicles 2018;7:1535750.

17. Mansouri A, Gattolliat CH, Asselah T. Mitochondrial Dysfunction and Signaling in Chronic Liver Diseases. Gastroenterology 2018;155:629-47.

18. Simonsen JB. Pitfalls associated with lipophilic fluorophore staining of extracellular vesicles for uptake studies. J Extracell Vesicles 2019;8:1582237.

19. Gupta D, Zickler AM, El Andaloussi S. Dosing extracellular vesicles. Adv Drug Deliv Rev 2021;178:113961.

20. Crewe C, Joffin N, Rutkowski JM, et al. An Endothelial-
to-Adipocyte Extracellular Vesicle Axis Governed by Metabolic State. Cell 2018;175:695-708.e13.

21. Pisano A, Cerbelli B, Perli E, et al. Impaired mitochondrial biogenesis is a common feature to myocardial hypertrophy and end-stage ischemic heart failure. Cardiovasc Pathol 2016;25:103-12.

22. Gan L, Liu D, Xie D, et al. Ischemic Heart-Derived Small Extracellular Vesicles Impair Adipocyte Function. Circ Res 2022;130:48-66.

23. Newell-Fugate AE. The role of sex steroids in white adipose tissue adipocyte function. Reproduction 2017;153:R133-49.

doi: 10.21037/exrna-21-28

Cite this article as: Paget D, Akbar N. A matter of fat: protecting the injured heart. ExRNA 2022;4:5. 